\title{
Das European Centre for Refractories schafft Synergien
}

\author{
Am Standort Höhr-Grenzhausen im Westerwald vereint das integrierte europäische \\ Feuerfest-Zentrum ECREF Aspekte der Bildung, Forschung und Innovation an einem \\ Standort. Die Aufgabe des ECREF besteht darin, die Feuerfestindustrie bestmöglich zu \\ unterstützen, beispielsweise bei der Ausbildung von Nachwuchs oder auch bei der \\ Weiterbildung von Fachpersonal. Einen besonderen Stellenwert haben Forschungs- und \\ Entwicklungsprojekte gemeinsam mit Produzenten und Anwendern.
}

In der modernen Volkswirtschaft zählt die Technologie der feuerfesten Keramiken zu den strategischen Schlüsseltechnologien, ohne die eine auf Fertigungs- und Produktionstiefe ausgelegte Nationalökonomie nicht vorstellbar ist. Die Industriezweige, in denen feuerfeste Keramiken eingesetzt werden, erzielen dabei einen vielfachen Umsatz der Schlüsselindustrie Feuerfest selbst. Deutschland besitzt die größte Feuerfest produzierende Wirtschaftsbranche Europas (Umsatz ca. 1,5 Mrd. €/a; ca. 6.000 Beschäftigte) und eine der wichtigsten der Welt. Die thematische Ausrichtung der Branche Feuerfest endet dabei nicht bei der Herstellung von keramischen Bauteilen oder Werkstoffen. Sie erstreckt sich vielmehr auf die Bereiche der Rohstoffwirtschaft, der Fertigung von feuerfesten Erzeugnissen und der Ausbildung zu dem Wissen und Können, auch größte Anlagen und Thermoprozessräume mit den richtigen feuerfesten Systemen auszustatten.

Feuerfest ist heute ein global denkender und agierender Wirtschaftszweig, in dem die Akteure hoch vernetzt sind. Die überwiegende Anzahl der in Deutschland operierenden Feuerfest-Produzenten und ihrer Zulieferer sind dabei im Verband der Deutschen Feuerfest-Industrie e. V. (www.vdffi.de) zusammengefasst. In dieser Konstellation hat sich im nördlichen Rheinland-Pfalz ein europaweit einmaliges Kompetenzzentrum der feuerfesten Keramiken ausgebildet. Am Standort Höhr-Grenzhausen im Westerwald systematisiert das integrierte europäische Feuerfest-Zentrum ECREF (European Centre for Refractories) die Synergie zwischen Wirtschaft und Wissenschaft. Das European
Centre for Refractories verbindet dabei die Aspekte der Bildung, Forschung und Innovation an einem Standort (Bild 1). Diese drei Aspekte werden integriert gedacht und gelebt, indem Bildung die Grundlage für Forschung legt und Forschung die Grundlage für Innovation. Für die Feuerfestindustrie, deren Produkte in Industriezweigen mit Hochtemperaturanwendungen verwendet werden, deren Prozesse ständig verbessert oder (wie in der Stahlerzeugung) auch komplett verändert werden, sind Bildung, Forschung und Innovation von besonderer Bedeutung. Die Aufgabe des ECREF besteht darin, die Feuerfestindustrie in diesen Aspekten bestmöglich zu unterstützen.

\section{Aus- und Weiterbildung}

Zur Ausbildung junger Menschen, die in der Feuerfestindustrie tätig sein wollen, wurde IRES (Integrated Refractory Education System) entwickelt und umgesetzt. Es umfasst Berufs- sowie Fachschulen, Hochschulen und Universitäten in der Region um Höhr-Grenzhausen und stellt sicher, dass Wissen über Feuerfest zum Curriculum jeder keramischen Ausbildung gehört. IRES begleitet dabei Schulabgänger auf jeder Ebene der beruflichen Bildung und Qualifikation: Von der Ausbildung (Berufsbildende Schule, Montabaur) über die Qualifikation zum Keramiktechniker (Staatliche Fachschule für Keramik, Höhr-Grenz-

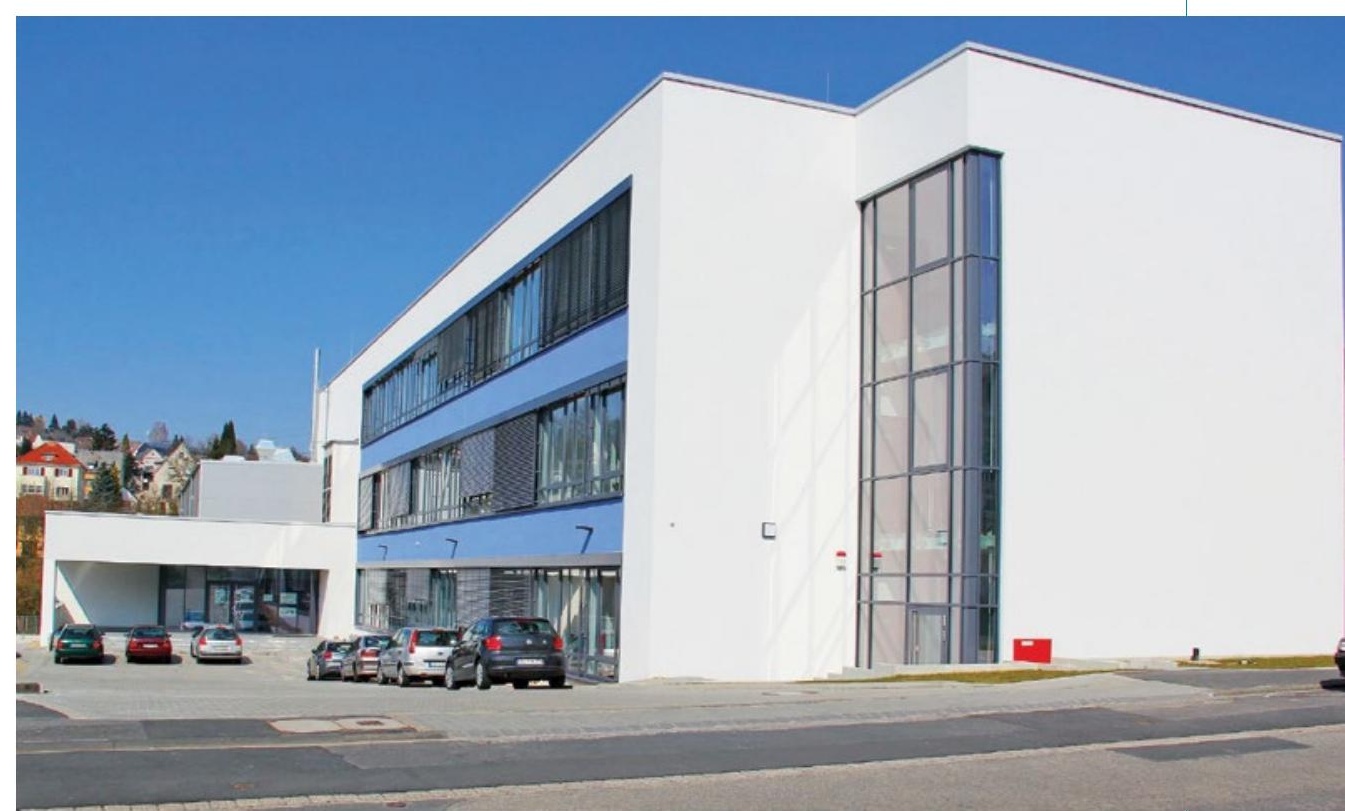

Bild 1 Integriertes europäisches Feuerfest-Zentrum (ECREF) in Höhr-Grenzhausen (๑ ECREF) 
hausen) über die akademische Bildung (Hochschule Koblenz, Höhr-Grenzhausen) bis zur Promotion (Universität KoblenzLandau, Koblenz).

In der Zusammenarbeit zwischen der Hochschule Koblenz und der Universität Koblenz-Landau wird einer der ersten gemeinsamen Master-Studiengänge angeboten (Master of Ceramic Science and Engineering), in den jede der beiden Einrichtungen ihre Erfahrungen und Schwerpunkte der Lehre von Feuerfest einbringt. Im Rahmen eines kooperativen Forschungskollegs „Maxvon-Laue Institute of Advanced Ceramic Material Properties Studies" der Universität Koblenz-Landau und der Hochschule Koblenz werden mittels eines internationalen und interdisziplinären Ansatzes zudem Doktoranden über den Zeitraum ihrer Promotion stetig weitergebildet. Dergestalt wird gleichzeitig eine gesellschaftliche sowie regionale Netzwerkbildung angeregt. Die Bildung und Forschung der Universität Koblenz im Bereich Feuerfest wird darüber hinaus von der Alexander Tutsek Stiftung geför-

Bild 2 Prinzip der HochtemperaturTemperaturwechsel-Prüfeinrichtung der FGF (๑ FGF)

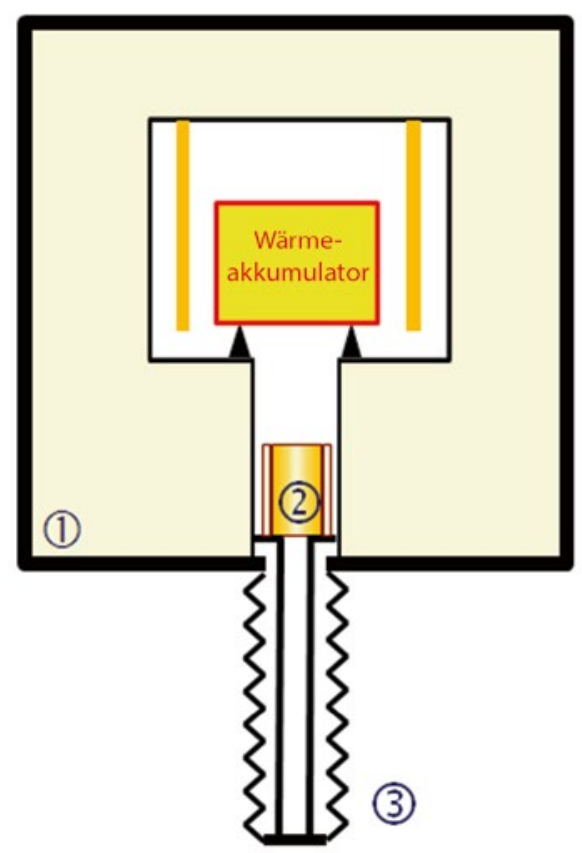

dert, die durch Preisgelder und Stipendien Nachwuchstalente finanziert.

Über die Ausbildung hinaus unterstützt ECREF die Weiterbildung von Personal der Feuerfestindustrie, ihrer Zulieferer und ihrer Kunden. Als Einführung in alle Bereiche von Feuerfest, insbesondere auch in die Verwendung von Feuerfest in den einzelnen Nutzerindustrien, wird jährlich das dreitägige Seminar „Feuerfest - Schlüsseltechnologie und ihre Anwendungen" abgehalten - in Kooperation mit dem europäischen Industrieverband der Feuerfestindustrie, PRE, auch jedes zweite Jahr in englischer Sprache. Wechselnde Industrieseminare zu Themen der Prozesstechnik (Brennen, Pressen, Mischen...) ergänzen das Seminarangebot. Zur kontinuierlichen wissenschaftlichen Weiterbildung und als Leitmesse der europäischen Feuerfestindustrie, organisiert ECREF das jährliche „Internationale Feuerfest-Kolloquium (ICR, International Colloquium on Refractories), das in 2020 bedingt durch COVID-19 auf ein virtuelles Format umgestellt wird.

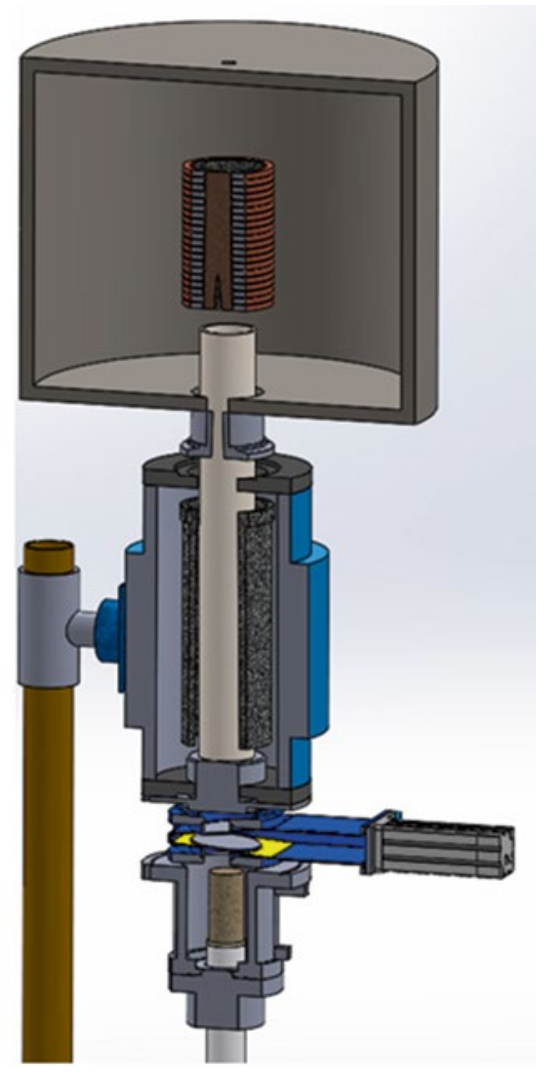

\section{Forschung}

Bei Forschung und Innovation hat die konsequente Unterstützung von Feuerfestproduzenten und -Anwendern einen besonderen Stellenwert. Im integrierten europäischen Feuerfest-Zentrum in Höhr-Grenzhausen führt die Forschungsgemeinschaft Feuerfest e. V. (FGF) zusammen mit Feuerfestproduzenten und Feuerfestanwendern Forschungs- und Entwicklungsprojekte durch. Insbesondere durch die Vernetzung mit Anwenderindustrien, die feuerfeste Werkstoffe einsetzen, ist die Forschung und Entwicklung anwendungs- und ergebnisorientiert. Kennzeichnend für die Arbeit der FGF ist zudem eine große Bandbreite von grundlagenforschungsorientierter, öffentlich geförderter Gemeinschaftsforschung zwischen Produzenten und Anwendern (z. B. im Forschungsrahmenprogramm der Europäischen Gemeinschaft) bis zu bilateralen Entwicklungskooperationen auf vertraulicher Basis.

Ein Arbeitsschwerpunkt der Forschungsgemeinschaft Feuerfest e. V. liegt in der Entwicklung von Hochtemperatur-Materialprüfmethoden, die über die vorhandenen Prüfsysteme weit hinausgehen. Die immer höheren Anforderungen an feuerfeste Keramiken in den Anwenderindustrien, bei denen hohe Temperaturen, hohe mechanische Belastungen und korrosive Atmosphären zusammenkommen, verlangen neue Werkstoffe, die auf diese Anforderungen hin gezielt entwickelt werden müssen. Aussagekräftige Hochtemperatur-Materialprüfmethoden für thermophysikalische und thermomechanische Eigenschaften tragen dabei entscheidend zur Leistungsfähigkeit von feuerfesten Keramiken bei. Sie liefern zudem wertvolle Materialkennwerte zur mathematischen Simulation und Optimierung des Verhaltens feuerfester Bauteile mittels FEM, die die Werkstoffentwicklung ergänzen.

\section{Beispiel: Temperaturwech- selbeständigkeit}

Im Zuge der Energieoptimierung werden viele industrielle Prozesse zunehmend dynamischer betrieben. In solchen Fällen werden die dort eingesetzten feuerfesten Werkstoffe stärker als bislang mit Temperaturwechseln belastet. Um das Verhalten von feuerfesten 
Materialien bei dynamischen Temperaturwechseln bei hohen Temperaturen zu untersuchen, hat die FGF daher eine Hochtemperatur-Temperaturwechsel-Prüfeinrichtung entwickelt (Bild 2), bei der Probekörper zwischen verschiedenen Temperaturen, beispielsweise $1000^{\circ} \mathrm{C}$ und $1600{ }^{\circ} \mathrm{C}$, zykliert werden können. Der Energieeintrag ist dabei anwendungsnah gestaltet und ist früheren Systemen deutlich überlegen. Die Ergebnisse solcher Prüfungen, bei denen die Schädigung der Probekörper zukünftig auch in situ gemessen werden kann, unterstützen die zielgerichtete Entwicklung verbesserter feuerfester Werkstoffe in der Feuerfestindustrie.

\section{Beispiel: Zementindustrie}

Zur Verbesserung feuerfester Keramiken für den Einsatz in der Zementindustrie und für Untersuchungen der Wechselwirkungen zwischen Sekundärbrennstoffaschen und feuerfesten Auskleidungen kommt bei der FGF ein Labor-Drehtrommelofen zum Einsatz, der mit einem speziellen Erdgas-Sauerstoff-Brenner ausgestattet ist (Bild 3). Mit diesem Hochleistungsbrenner können im Labor die hohen Temperaturen und die Atmosphären erreicht werden, die in industriellen Zementdrehrohröfen vorherrschen.

\section{Beispiel: Energiewende}

Aspekte der Energiewende, sei es die Verwendung von Biogas und Wasserstoff als Ersatz für Erdgas oder die Umstellung auf Strom, verlangen von der Feuerfestindustrie angepasste bis vollkommen veränderte Brennprozesse. Die FGF betreibt dazu Studien, thermochemische Simulationen ( $\mathrm{z}$. B. mittels Factsage), experimentelle Untersuchungen, Werkstoffanalysen und standardi-

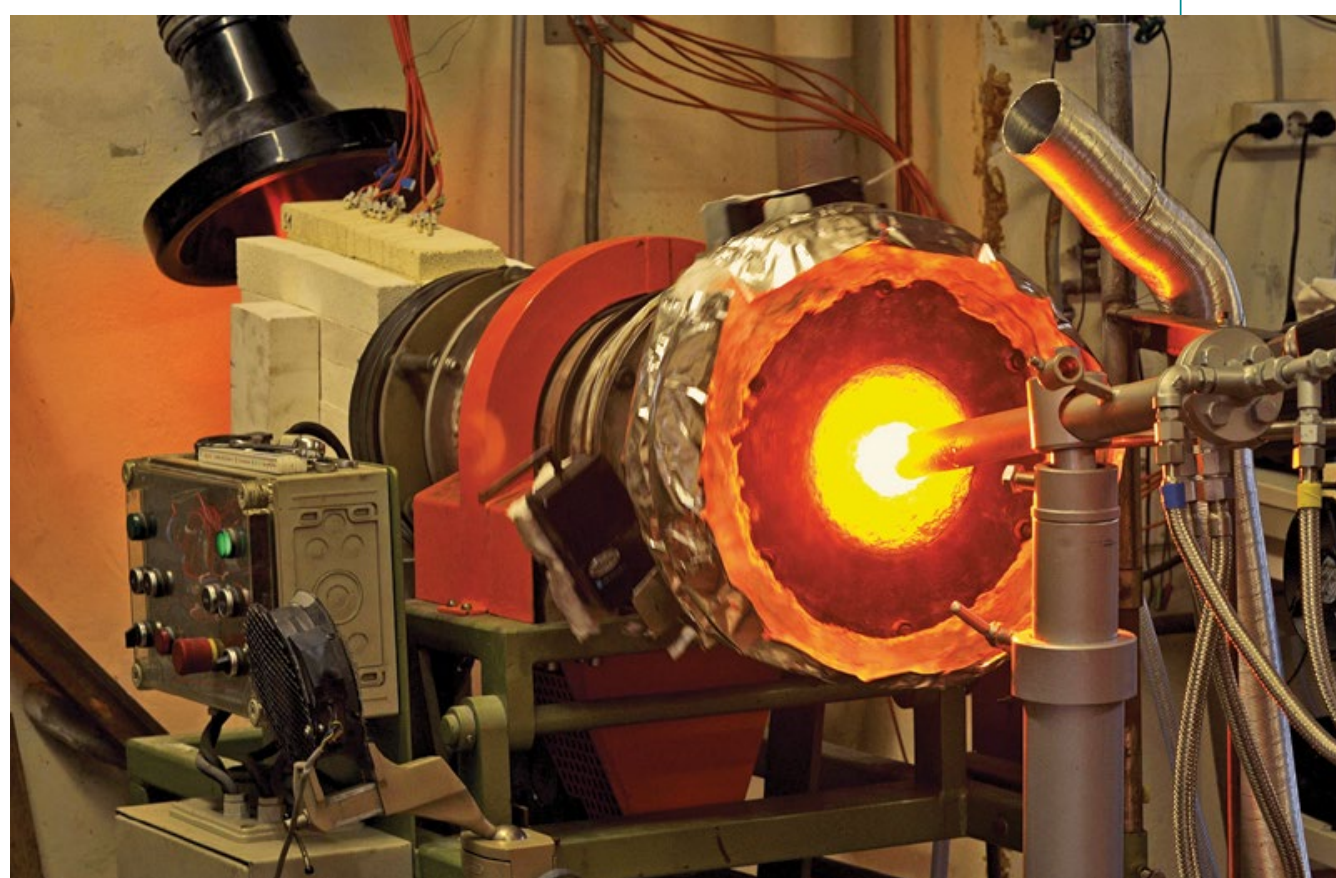

Bild 3 Drehtrommelofen für Klinkerbrand mit Zuführung von Sekundärbrennstoffaschen (৫ FGF)

sierte Vergleichsmessungen zur Untersuchung des Einflusses des Energieträgers beim Brand auf die Qualität feuerfester Rohstoffe und Erzeugnisse.

\section{Innovation}

Innovation, also die Entwicklung und Produktion von neuen und verbesserten feuerfesten Erzeugnissen, ist Kernaufgabe der Feuerfestindustrie. Eine wichtige Voraussetzung dafür ist der Transfer von neuem Wissen aus der Forschung in die Industrie. ECREF unterstützt diesen Transfer auf möglichst kurzem Wege, indem Forschung (FGF) und Industrie (VDFFI) unmittelbar zusammenarbeiten. Dies manifestiert sich in gemeinsamen Arbeitsausschüssen oder in Forschungsprojekten der industriellen Gemeinschaftsforschung (Programm IGF des BMWi), bei denen bereits während der Projektlaufzeit regelmäßige Transfersitzungen stattfinden.

Bei der Umsetzung von Innovationen wird die Feuerfestindustrie darüber hinaus vom im ECREF angesiedelten DIFK - Deutschen Institut für Feuerfest und Keramik $\mathrm{GmbH}$ in jeder Hinsicht unterstützt. Das DIFK bietet als unabhängiges, akkreditiertes Prüflabor ein außerordentliches Angebot an Materialprüfungen an. Damit werden insbesondere kleine und mittlere Unternehmen der Feuerfestindustrie in die Lage versetzt, ihre Produkte ohne eigene Laborkapazitäten zu prüfen und zu verbessern.

Kontakt:

European Centre for Refractories gGmbH,

Höhr-Grenzhausen, www.ecref.eu

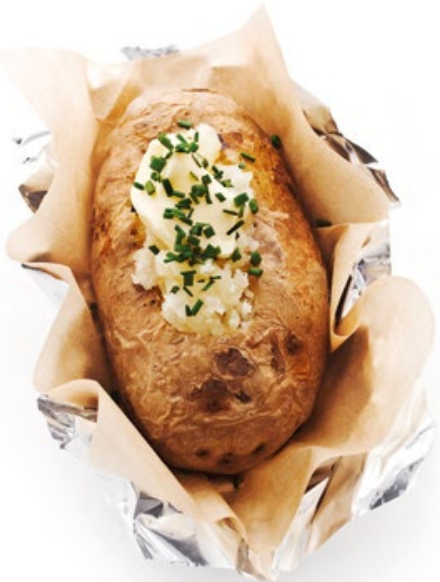

\section{Flexibler Schutz für heiße Rundungen}

\section{Fasertechnische Halbzeuge für Hochtemperatur-Anwendungen}

$\nabla$ Biolösliche Keramikfaser-Produkte (Alcaline-Earth-Silicate) für bis zu $1260^{\circ} \mathrm{C}$

$\nabla$ Bindemittelfreie keramische Aluminiumsilikat-Halbzeuge für bis zu $1500^{\circ} \mathrm{C}$

$\nabla$ Polykristalline Aluminiumoxidwollen für bis zu $1850^{\circ} \mathrm{C}$

$\nabla$ Konventionelle Keramikfaser-Produkte für bis zu $1250^{\circ} \mathrm{C}$

$\boldsymbol{\nabla}$ Flexible Halbzeuge zum Isolieren, Dämmen, Schützen, Aus- und Verkleiden

$\boldsymbol{\nabla}$ Ideal für Ofentechnik, Motorenbau, Feuerfest-Industrie, Hochvakuumtechnik u.a.

$\boldsymbol{\nabla}$ Lieferbar als Papiere, Vliese, Filze, Platten, Füllstoffe, Knetmassen und Formteile

Hochtemperaturprodukte | Kälteerzeugung | Dichten und Kleben | Messtechnik | Beschichtungen | Problemlöser

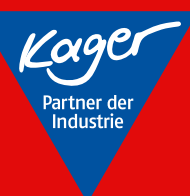

Paul-Ehrlich-Str. 10a

D-63128 Dietzenbach

Tel. +49-(0)6074-40093-0

info@kager.de

www.kager.de

Zertifiziert nach

DIN EN ISO 9001:2015 